EGU2020-16122

https://doi.org/10.5194/egusphere-egu2020-16122

EGU General Assembly 2020

(c) Author(s) 2020. This work is distributed under

the Creative Commons Attribution 4.0 License.

\title{
TWIGA project activities for the enhancement of heavy rainfall predictions in Africa: low-cost GNSS network deployment and NWP model parameterization.
}

\author{
Alessandra Mascitelli ${ }^{1}$, Agostino Niyonkuru Meroni ${ }^{1}$, Stefano Barindelli ${ }^{1}$, Marco Manzoni ${ }^{2}$, Giulio \\ Tagliaferro ${ }^{1,3}$, Andrea Gatti ${ }^{3}$, Eugenio Realini ${ }^{3}$, Giovanna Venuti ${ }^{1}$, and Andrea Monti Guarnieri ${ }^{2}$ \\ ${ }^{1}$ DICA, Politecnico di Milano, Milano, (giovanna.venuti@polimi.it) \\ ${ }^{2}$ DEIB, Politecnico di Milano, Milano \\ ${ }^{3}$ Geomatics Research and Development s.r.l., Geodesy and Geomatics, Lomazzo, Italy (eugenio.realini@g-red.eu)
}

One of the objectives of the H2020 project TWIGA - Transforming Weather Water data into valueadded Information services for sustainable Growth in Africa - is the improvement of heavy rainfall prediction in Africa. In this area, the scarcity of data to support such predictions makes it fundamental to enhance the monitoring of atmospheric parameters.

In this project, GNSS observations and SAR images from Sentinel missions are used to produce water vapor products to be assimilated into Numerical Weather Prediction Models (NWPS).

GNSS observations, collected by ad-hoc networks of geodetic and low-cost stations, are processed to obtain near real-time (NRT) Zenith Total Delay (ZTD) time series, while Sentinel-1 SAR images are used to derive Atmospheric Phase Screens, APSs. The free and open source GNSS software goGPS, developed by the Politecnico di Milano spin-off Geomatics Research and Development (GReD), is used for the retrieval of ZTDs time series.

After proper calibration and validation procedures, the delay maps from SAR and the delay time series from GNSS will be finally assimilated into NWP models to improve the prediction of heavy rainfall.

The GNSS-related activities will be presented in terms of network deployment and processing settings evaluation. A network of 5 single-frequency low-cost GNSS stations was installed in Uganda, and a new network of dual-frequency low-cost stations is going to be installed in Kenya. To improve the outputs provided by these networks, preliminary tests on ionospheric delay corrections at various distances were performed. Different methods, focused on the reconstruction of a synthetic L2 observation for the single-frequency receivers, were employed and evaluated with the aim to define the optimal approach.

In order to demonstrate the capability to achieve GNSS NRT processing within TWIGA, an automated procedure was set up to estimate hourly ZTDs from two geodetic permanent stations located in South Africa (Cape Town and Southerland) and to upload them to the TWIGA project 
web portal.

Meanwhile, first sets of WRF NWP model parameterizations have been defined for both South Africa and Kenya. A cooperation has been established with the Kenya Meteorological Department on the exploitation of 3DVAR tool for water vapor data assimilation into WRF. Studies to define a strategy for ZTD maps retrieval from InSAR APS have been performed on Italian datasets and further investigations on TWIGA-collected African datasets will follow.

How to cite: Mascitelli, A., Meroni, A. N., Barindelli, S., Manzoni, M., Tagliaferro, G., Gatti, A., Realini, E., Venuti, G., and Monti Guarnieri, A.: TWIGA project activities for the enhancement of heavy rainfall predictions in Africa: low-cost GNSS network deployment and NWP model parameterization., EGU General Assembly 2020, Online, 4-8 May 2020, EGU2020-16122, https://doi.org/10.5194/egusphere-egu2020-16122, 2020 